made by a skilful mechanic it could take the form of a faultless, silently running and exact instrument. It could even be further improved for scientific purposes. It would be necessary, for example, to make its illumination independent of daylight; to narrow the field of observation to points and to bring them together into one round divided surface, observed through a telescope, similar to that of Nagel's anomaloscope. For the present I had to content myself with the model at hand.

\title{
A NEW METHOD OF APPLYING THE SCREEN TEST FOR INTER-OCULAR MUSCLE BALANCE
}

BY

\section{T. LEES}

ASSISTANT REGISTRAR, MANCHESTER ROYAL EYE HOSPITAL

THE apparatus described in this communication was developed at the Manchester Royal Eye Hospital as the result of a series of experiments designed to overcome certain difficulties experienced in the application of the Hess screen test.

In the Hess method, a colour separation system is used to obtain dissociation of binocular vision, and the difficulties experienced were all attributed to this. Such a system, whilst theoretically capable of perfect results, is apt to be inefficient in practice, because of the difficulty of manufacturing materials of the exact and stable colours required for the screen, pointer, and goggles. Even if initially correct, these are likely to deteriorate with age and use. If the colours of the components used are not exactly correct, the dissociation obtained is only of relative degree, and errors then are liable to occur in the test. Thus the red pattern of the screen may be faintly visible through the green glass, or the green pointer through the red glass. The resultant accuracy of the test depends on the brightness of these images compared to the correct ones.

If the secondary image allows of uniocular localization of the pointer to the cardinal points through either glass, the test is rendered useless; or even if the position of the green pointer is seen through the green glass, and related, as it should be, to the apparent position of the red cardinal points seen through the red glass, still the result may be inaccurate, owing to the fusional stimulus caused by the screen pattern being visible, to a greater 
or lesser degree, to each eye, for this distorts the possible angle of deviation.

Even when colour separation is perfect, errors may still occur, for a keen observer may be able to effect monocular localization of the pointer to the cardinal points if he notices the eclipse of the red screen-pattern which takes place as the pointer is moved over its surface.

Many patients have trouble with the test for yet another reason, as they fail to recognize the pattern of the screen, or the position of the pointer, because of the low level of contrast present, when these are seen against the black background of the screen through red-green goggles. As a result, they cannot appreciate what is required of them to complete the test. This inability is partly due in a small proportion of cases to some defect of red-green vision in the patient under test.

In order to obviate the possibility of such errors and difficulties, and to make the screen test more easy to perform, it was redesigned around a more efficient and stable method of dissociation. The tangent scale pattern used on the Hess screen was retained, but as colour separation was not used, the scale was printed in black on a white background to ensure clarity. The Hess test recording charts are used as previously, and the graphs produced are interpreted in exactly the same manner as before.

The principle of this new development of the screen test is that dissociation is obtained by a plane mirror placed before one eye. The mirror has a reflecting surface on each side, and is placed in such a position between two identically printed tangent scales erected at right-angles to each other, that a symmetrical system is formed, in which each scale is situated at the position to which the virtual image of the other scale is projected, when seen in one or the other surface of the mirror. Thus the patient sees different but identical scales exactly superimposed. To avoid fusional stimulus and afford a blank surface before the plotting eye, each scale is so constructed that its pattern may be made to appear or disappear as required during the test.

Reference to the illustrations .will amplify the following description of the construction and use of the apparatus.

Two tangent screens are used, each being a composite assembly of a plotting screen and tangent scale. Each plotting screen consists of a sheet of light opal perspex, 32 in. square $x \frac{1}{8}$ in., mounted on the top half of a wooden stand, 64 in. in height. Behind each screen is a tangent scale, $30 \mathrm{in}$. square, the pattern of which is exactly as is used on the Hess screen, that is, one having a working distance of $50 \mathrm{~cm}$. It is, however, marked in black lines on a white background. The tangent scale is sup- 


\section{PLAN OF MIRROR SCREEN TEST}
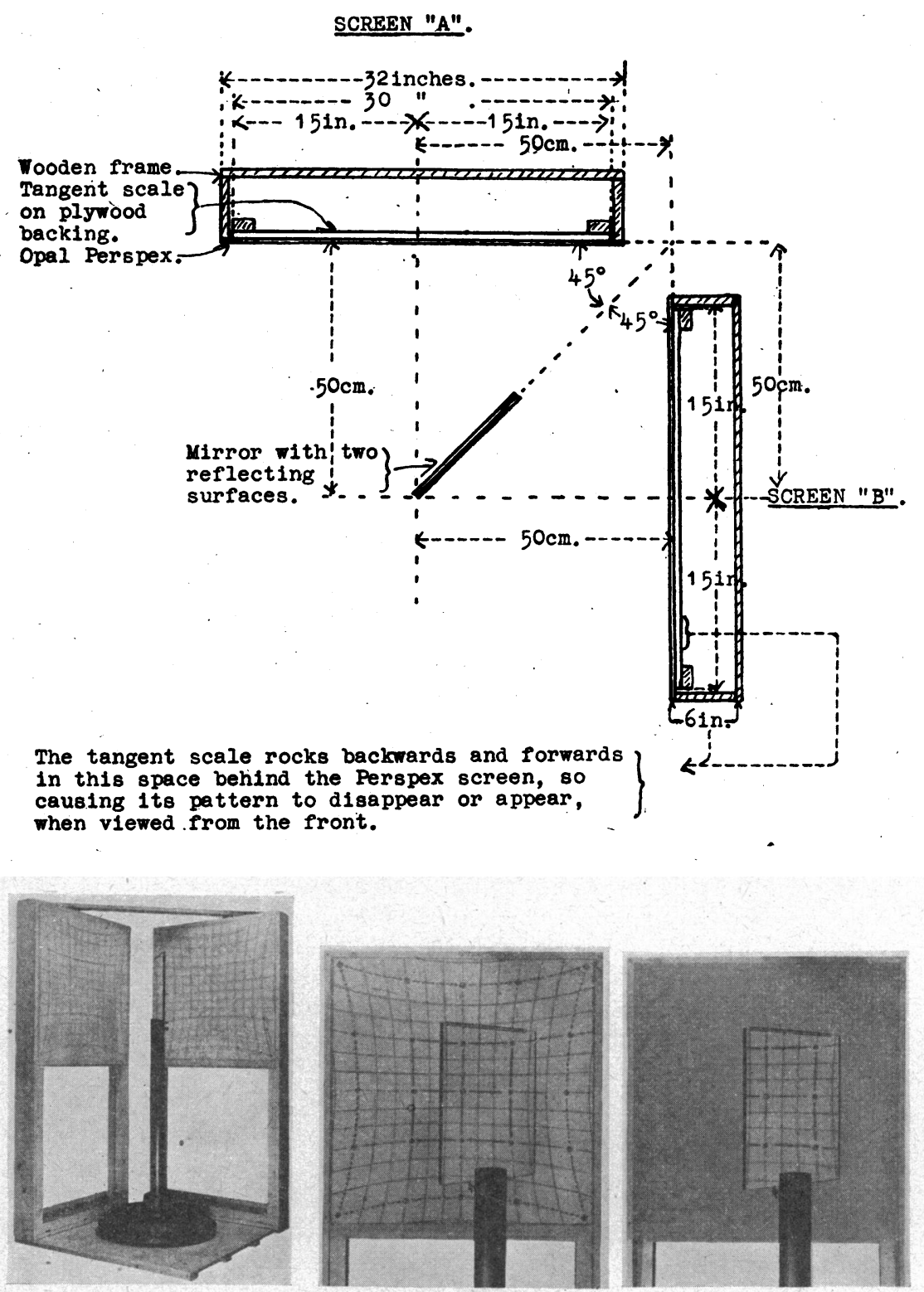

(Photographs by courtesy of the Defartment of Medical Photografly. Yanchester Royal In fir muary)

General view of apparatus. Screen "A" on the left. Screen " $B$ " on the right.
Screen "A" with virtual image of screen "B" seen superimposed in the mirror.
Screen "A" with tangent pattern suppressed ready for plotting projection of left eye. The right eye fixing the mirror image. 
ported by a plywood back-board held on two wooden struts, which hinge on to the bottom of the frame holding the plotting screen. The tangent scale is thereby enabled to rock backwards and forwards behind the plotting screen, so as to be either in contact with it, when its pattern shows clearly through the opal perspex, or separated from it by a distance of about 4 in., when its pattern is so diffused as to be invisible.

The two tangent screen assemblies are secured in relation to each other on a firm baseboard, being also braced by a top strut. Their position is such that the two plotting screens are vertical and at right-angles to one another, and so that with both tangent patterns made visible, the centre horizontal lines of the scales are each at the same height from the base, and the centre vertical lines each parallel to, and $50 \mathrm{~cm}$. distant from, the plane of the other screen. These adjustments must be accurately carried out, as upon them and the position of the mirror, rest the exact alignment required for the success of the test.

The mirror is made from two $\frac{1}{8}$ in. thick sheets of plate-glass, each 12 in. square, silvered on the rear surface and cemented together back to back so as to give a reflecting surface on each side. This mirror is mounted on a rigid steel stand equipped with screwed bolts in the base, whereby the position of the mirror can be adjusted.

The mirror on its stand is placed in position between the plotting screens, so that its reflecting surfaces lie in the plane which bisects the angle between them-that is, at angle of 45 degrees to either screen. The front edge of the mirror must be on a line 50 $\mathrm{cm}$. distant on perpendiculars drawn from the centre verticals of either tangent screen. The height of-the mirror should be such that the line bisecting it horizontally is level with the centre horizontals of the tangent screens.

- The accuracy of the alignments carried out may now be checked by viewing each screen, and the part of the other screen as seen in the mirror, from a distance of about $6 \mathrm{ft}$., when the lines of the virtual image should. be continuous with those of the screen on which it is superimposed. If this is found to be correct the apparatus may be considered ready for use.

Two pointers are used in the test, each consisting of a metal rod about $50 \mathrm{~cm}$. long. The one used by the patient has a ring at its tip after the style of the Law pointer often used in the Hess test. The one used by the operator has a small disc at its tip half the diameter of the ring on the other.

The patient sits close to the mirror, with his nose touching its front edge. He faces scteen "A" if the projection of his left eye is to be tested, or screen " B" if his right is to be tested. 
The charts obtained by plotting from these positions correspond to those obtained by the Hess method when wearing the green glass before the left and right eyes respectively.

Suppose the patient to be facing screen "A." His right vision is intercepted by the mirror, in which he sees screen " $B$ " projected forwards as a virtual image, to appear as if superimposed upon screen "A." The patient's left eye, its vision unobstructed by the mirror, sees screen "A" directly, but cannot see screen " $B$ ". as this is situated laterally. Nor can the right eye see screen " $A$ " because of the mirror. Binocular vision is, therefore, completely dissociated, but similar tangent scales are exactly superimposed by the symmetry of the apparatus.

The tangent pattern of screen " A " is now suppressed, leaving a blank surface before the patient's left eye. The tangent pattern of screen "B" still remains visible to the patient's right eye, appearing as a virtual image projected on to the blank surface of "A." The operator now indicates on screen " $B$ " the cardinal points which he desires the patient to fix, by holding the disc at the tip of his pointer over each position in turn. Small oscillatory movements are made with the disc over the cardinal point, so as to stimulate more exact foveal fixation by the patient, who observes the virtual image of the disc through the mirror. These movements also help to eliminate alternating macular suppression, which sometimes causes trouble during the test. The patient holds the ring-tipped pointer in his left hand and with it encircles the apparent position of the disc. As the ring is seen only by his left eye against the blank screen "A," it indicates the foveal projection by this eye of the image of the disc seen by his right eye, depending upon the cerebral relation of the two foveal pathways, and the interocular muscle balance. The position of the ring is now measured by momentarily causing the re-appearance of the tangent pattern on screen "A."

The cardinal points required to complete the square pattern covering the ocular muscle actions are now plotted in sequence. The measurements taken are entered on the left-hand side of the diagnosis chart, exactly as plotted by the patient on screen "A."

It will be realized that the patient sees the virtual tangent scale reversed by the mirror in the horizontal meridian, but as the nine cardinal points are symmetrically situated about the centre line, the phenomenon of image reversal need not be taken into account when all nine points are plotted in sequence. Only if a single point is under consideration during plotting need this reversal effect be remembered.

To test the right projection, the patient must change his position so as to face screen " $B$. ." This time he fixes the cardinal 
points indicated on screen "A" with his left eye, which now confronts the other reflecting surface of the mirror. The projections of the visual axis of the right eye corresponding to the various points fixed by the left, are now indicated by the patient, who this time holds his pointer in his right hand and guides it by the vision of his right eye over screen " $B$." During this half of the test the tangent pattern of screen " $B$ " is suppressed during plotting.

The measurements taken are transferred to the right-hand side of the diagnosis chart, which on completion is interpreted in exactly the same manner as is a record of the test done by the red-green dissociation technique of Hess.

The method of construction described above is not the only one applicable to the screen test by mirror dissociation, but was one adopted for the sake of convenience and according to the workshop facilities at our disposal.

Possibly a better method of superimposing the tangent patterns on to the plotting screen is a rear-projection system, in which the image of a tangent scale printed on a transparent base, is thrown on to an opal screen by back-lighting. By this means control of the pattern is obtained through an electric switch. Messrs. Theodore Hamblin, Ltd., of Wigmore Street, London, are conducting experiments on this system with a view to production of the apparatus.

Another way is to employ symmetrically mounted cork or suchlike screens, on which the plotting of the patient's projection is mapped by pins. The cardinal points for fixation are shown by a transparent tangent scale, which, on removal to the mirror image position, is also used to measure the position of the plotting pins.

Other variations, such as an automatic recording system, by which the projection is transferred directly to the graph-paper, are possibilities which have not yet been tried.

Whichever method is adopted, it will be realized that, as the apparatus requires to be installed as a semi-permanent fixture, and as its manufacture is more exacting, the initial cost will far exceed that for the original Hess screen test. The advantages offered in rapid, accurate and trouble-free results far outweigh the initial difficulties and expense involved.

The apparatus has been in daily use at the hospital at Manchester for almost a year, and during this time many hundreds of patients have been tested upon it. Although many of these had previously failed to perform the Hess test, not one failed to perform the mirror disscciation method. Amongst the numbers tested were several small children, all of whom readily gave readings which could be interpreted with confidence in their accuracy. 